\title{
Primary osteosarcoma of the urinary bladder treated with external radiotherapy in a patient with a history of transitional cell carcinoma: a case report
}

\author{
Christos Papandreou ${ }^{1 *}$, Antigoni Skopelitou², George Kappes ${ }^{3}$, Housam Daouaher ${ }^{1}$
}

\begin{abstract}
Introduction: Primary osteosarcoma is one of the rare tumors affecting the urinary bladder. The occurrence of osteosarcoma in a patient with a long history of transitional cell carcinoma of the bladder is even more uncommon.

Case presentation: We present the case of a 74-year-old Greek man who was diagnosed with osteosarcoma 10 years after he had been diagnosed with transitional cell carcinoma of the bladder from which he had been free from recurrences for the past three years. Our patient was treated for the osteosarcoma with transurethral resection of bladder tumor and external beam radiation therapy. He died eight months after the diagnosis, suffering poor quality of life in the last months.

Conclusion: Osteosarcoma of the bladder has a dismal prognosis. External beam radiation therapy as an adjunct to transurethral resection of bladder tumor not only provides no benefit to patients with primary osteosarcoma of urinary bladder, but also may be associated with poor quality of life.
\end{abstract}

\section{Introduction}

Osteosarcoma of the urinary bladder is a rare tumor. To date, only 32 cases have been reported using several treatment modalities [1-3]. We present another case of this neoplasm, which was managed with transurethral resection and external beam radiation therapy.

\section{Case presentation}

A 74-year-old Greek man presented in June 2008 with gross hematuria. Our patient had been previously treated for non-muscle invasive pTa, G2 transitional cell carcinoma of the bladder (TCC). The first transurethral resection of bladder tumour (TURBT) had been performed ten years before, while the last was performed three years before. The last follow up cystoscopy had been performed twelve months before. Our patient was asymptomatic thereafter, until June 2008.

\footnotetext{
* Correspondence: cpapas@otenet.gr

1 Department of Urology, General Hospital of Arta, Greece
}

Then, at cystoscopy, a large solitary polypoid, yellowwhite tumor was found on the superior surface of the bladder. A computed tomography (CT) scan revealed a heterogeneous mass arising from the bladder dome with evidence of invasion of the bladder wall (figure 1). There was no evidence of pelvic or abdominal lymphadenopathy. Subsequent transurethral resection was extremely difficult and incomplete because of hardness of the tissue. Pathological examination of retrieved specimen revealed a highly pleomorphic and hypercellular neoplasm composed of spindled sarcomatous cells with characteristic formation of malignant osteoid, as well as an abundant chondroid matrix.

Immunohistochemical analysis performed on paraffinembedded tissue sections was negative for pan-cytokeratin 7 and 20, epithelial membrane antigen (EMA), as well as a smooth muscle actin, desmin, CD34 and CD68. Vimentin and p53 were strongly expressed in more than $95 \%$ of the neoplasmatic cells. No transitional cell carcinoma or carcinosarcoma elements were evident (figures 2 and 3). These findings supported the diagnosis 


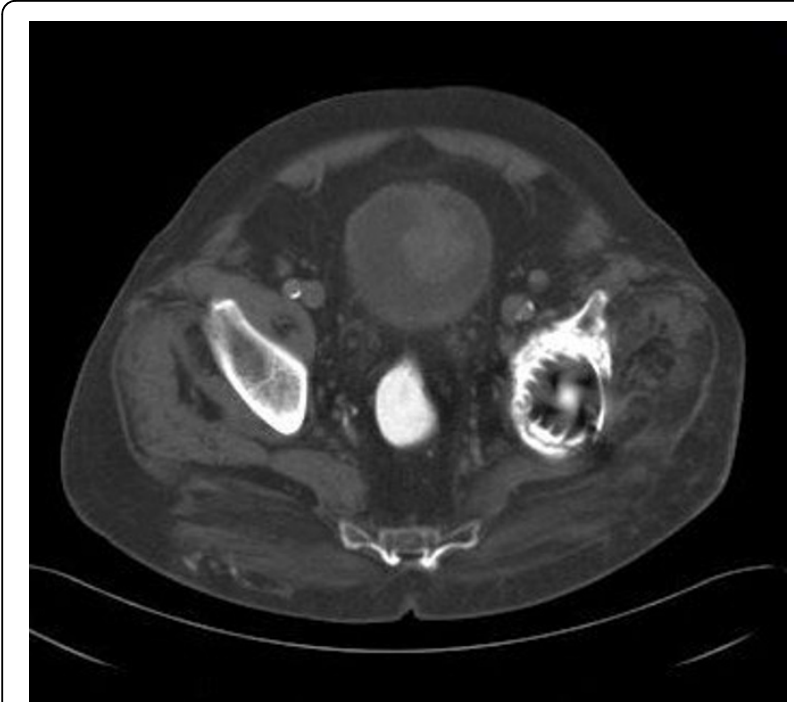

Figure 1 Computed tomography scan shows a large solitary polypoid tumour arising from the bladder dome. Also notice left hip arthroplasty.

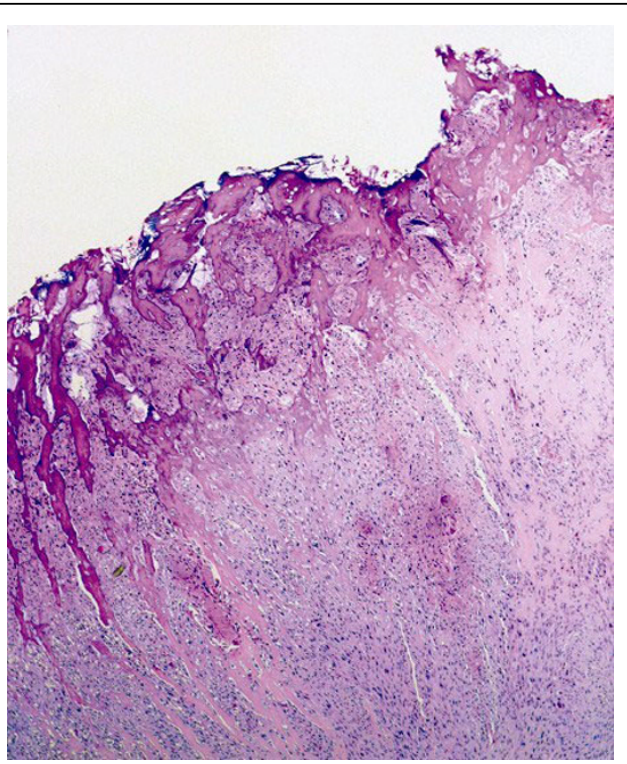

Figure 2 Cancellous bone and malignant, lace-like osteoid formation by the spindled sarcomatous cells and in between these cells chondroid matrix $(\mathrm{HE}, \times 100)$.

of primary osteogenic sarcoma of the urinary bladder. Other localization studies including a chest X-ray and a bone scan were unremarkable.

Due to the significant comorbidity of our patient, who had a coronary artery disease and restrictive respiratory disease, he was offered partial cystectomy. However, he refused this option and underwent external beam radiation therapy (EBRT) instead. Our patient declined to receive adjunctive chemotherapy. In the following

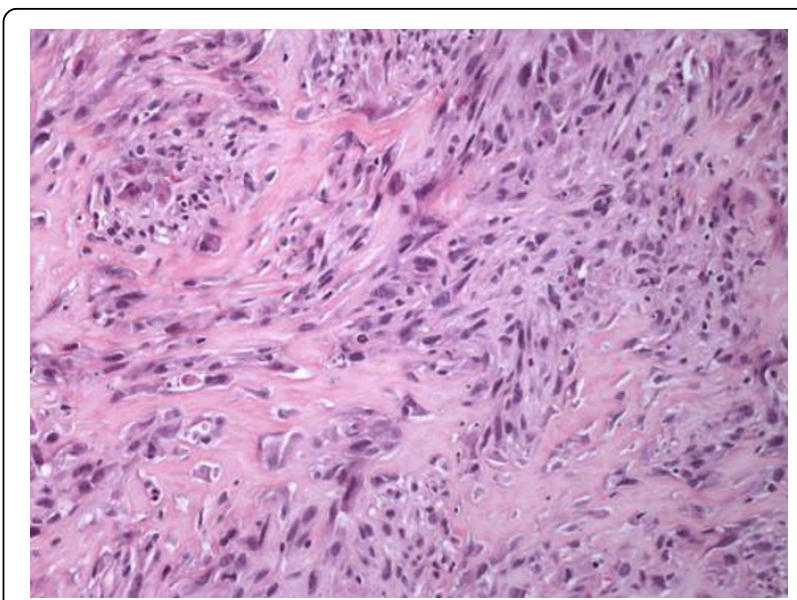

Figure 3 Malignant, lace-like osteoid formation. Notice the nuclear polymorphism of the malignant cells producing malignant woven bone. $(H E, \times 200)$

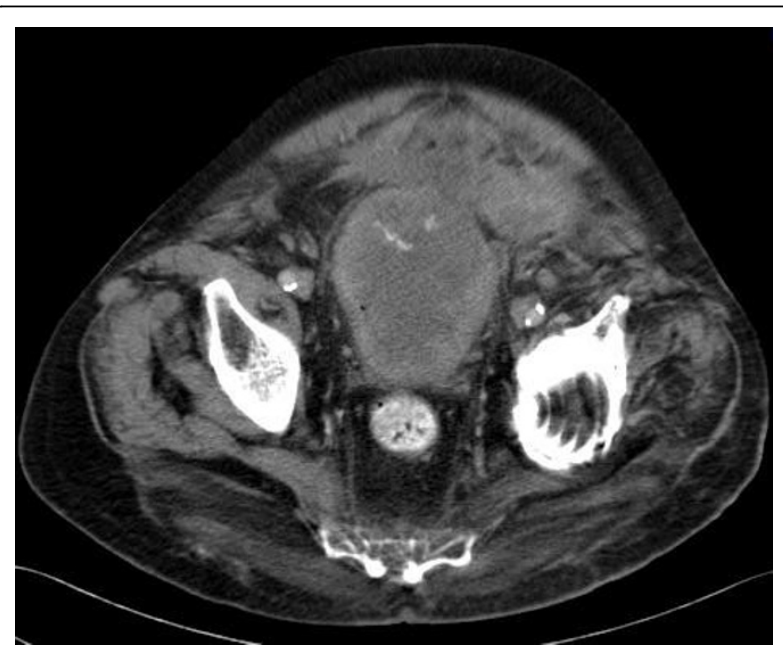

Figure 4 Computed tomography scan seven months afterdiagnosis shows extravesical tumour extension and bowel invasion.

months, he had two episodes of proven urinary tract infection. Seven months after diagnosis, he developed ascites. A CT scan at that point showed extravesical tumor extension and bowel infiltration (figure 4). Our patient died eight months after diagnosis, experiencing severe lower urinary tract symptoms (LUTS) and abdominal pain the last months of his life.

\section{Discussion}

Only 32 cases of primary osteosarcoma of the bladder have been so far reported in the English literature [1-3]. To the best of our knowledge, this is the fourth reported case where this type of tumor occurs in a patient with a history of TCC or simultaneously with TCC [4-6]. 
Osteosarcoma should be distinguished from other bone-forming tumors, such as carcinosarcoma and transitional cell carcinoma with osseous metaplasia. It is not always easy to distinguish extraskeletal osteosarcoma from other sarcomas, which rarely produce metaplastic bone such as malignant fibrous histiocytoma, synovial sarcoma and epithelioid sarcoma. In most of these neoplasms, osteoid or bone formation is confined in a small portion of the tumor and is relatively well differentiated, without the disorderly pattern and cellular/nuclear polymorphism/pleomorphism of osteosarcoma. The characteristic microscopic pattern of our tumor, that is, the malignant, lace-like osteoid, produced in large amounts by the spindled sarcomatous cells, and the presence of chondroid matrix between these cells along with the above-mentioned immunoprofile and the absence of concurrent epithelial malignancy, supported the diagnosis of primary osteosarcoma of urinary bladder [7-9].

The prognosis of bladder osteosarcoma is very poor, with most patients dying from the disease within six months. The tumor tends to be locally aggressive. Distant metastases are uncommon and are usually confined to the lung. Recommendations to improve survival have included radical cystectomy with radiotherapy and/or chemotherapy, but most patients die of this disease regardless of the type of treatment $[1,10]$.

However, there are publications that report successful treatment with a long follow- up. One patient was free of such disease 36 months after partial cystectomy [10] and another survived with no evidence of recurrence after 51 months of combination treatment with radical cystectomy and chemotherapy [11]. A case of bladder osteosarcoma with markedly remission of pulmonary metastases after chemotherapy has also been reported [1].

In our case, our patient had a long history of TCC and also had significant comorbidity. He was thoroughly informed about the disease and finally decided to undergo EBRT. Although he lived few months more than usual, during the last four months, he suffered severe LUTS, abdominal pain and was heavily medicated with opioid analgesics. For the above-mentioned symptoms, there was no obvious etiology other than radiotherapy.

\section{Conclusion}

Osteosarcoma of the bladder is a rare tumor with a dismal prognosis. External beam radiation therapy as adjunct to transurethral resection seems to provide no benefit for patients with primary osteosarcoma of urinary bladder. Moreover, it may be associated with poor quality of life and thus should be better to be avoided.

\section{Consent}

Written informed consent was obtained from our patient for publication of this case report and accompanying images. A copy of the written consent is available for review by the Editor-in-Chief of this journal.

\section{Abbreviations}

CT: Computed tomography; TCC: transitional cell carcinoma; EBRT: external beam radiation therapy; TURBT: transurethral resection of bladder tumor; LUTS: lower urinary tract symptoms.

\section{Author details}

'Department of Urology, General Hospital of Arta, Greece. ${ }^{2}$ Department of Pathology, General Hospital of Arta, Greece. ${ }^{3}$ Department of Radiology, General Hospital of Arta, Greece.

\section{Authors' contributions}

CP was involved in patient care, review of literature and writing of the manuscript. AS performed the histological examination and reviewed the literature. GK was involved in radiological evaluation of our patient. HD was involved in patient care and writing supervision. All authors read and approved the final manuscript.

\section{Competing interests}

The authors declare that they have no competing interests.

Received: 18 September 2009 Accepted: 24 February 2010 Published: 24 February 2010

\section{References}

1. Berenson RJ, Steven F, Freiha FS, Kempson RL, Torti FM: Primary osteogenic sarcoma of the bladder. Cancer 1986, 57:350-355.

2. Rodríguez Alonso A, González Blanco A, Bonelli Martín C, Nogueira Carballedo C, García Rego JA, Cachay Ayala M, Lorenzo Franco J, Cuerpo Pérez MA, Nieto García J: Primary bladder osteosarcoma treated with hemicystectomy. Actas Urol Esp 2002, 26:226-230.

3. Baydar DE, Himmetoglu C, Yazici S, Kiziloz H, Ozen H: Primary osteosarcoma of the urinary bladder following cyclophosphamide therapy for systemic lupus erythematosus: a case report. J Med Case Reports 2009, 3:39.

4. Carter RD, Powers $\mathrm{JH}$, van Hawn $\mathrm{CZ}$ : Osteogenic sarcoma and transitional cell carcinoma occurring simultaneously in the urinary bladder: report of a case. J Urol 1956, 76:263-269.

5. Abbas AM, Katz DS, Sommer FG: CT findings of primary osteosarcoma of the bladder. AJR 1996, 167:536-536.

6. Okada E, Hasegawa Y, Takahasi K, Goto K: Primary osteosarcoma of the urinary bladder. Int Urol Nephrol 1997, 29:437-440.

7. Young RH, Rosenberg AE: Osteosarcoma of the urinary bladder. Report of a case and review of the literature. Cancer 1987, 59:174-178.

8. Weiss SW, Goldblum JR: Osseous soft tissue tumours. Enzingers and Weiss's Soft Tissue Tumours Philadelphia: Mosby, 4 2001, 1405-1417.

9. Ghalayini IF, Bani-Hani IH, Almasri NM: Osteosarcoma of the urinary bladder occurring simultaneously with prostate and bowel carcinomas: report of a case and review of the literature. Arch Pathol Lab Med 2001, 125:793-795.

10. Kato T, Kubota Y, Saitu M, Yaguchi H, Sasagawa I, Nakada T, Yuda F: Osteosarcoma of the bladder successfully treated with partial cystectomy. J Urol 2000, 163:548-549.

11. Phan CN, Wilkinson M, Cohen MB, Dunn S, Carroll P: Primary osteogenic sarcoma of the urinary bladder successfully treated with combination therapy. Urology 1994, 44:771-774.

doi:10.1186/1752-1947-4-70

Cite this article as: Papandreou et al.: Primary osteosarcoma of the urinary bladder treated with external radiotherapy in a patient with a history of transitional cell carcinoma: a case report. Journal of Medical Case Reports 2010 4:70. 